\title{
Management of isolated coronal shear fractures of the humeral capitellum with Herbert screw fixation through anterolateral approach
}

\author{
Tengbo Yu ${ }^{1}$, Hao Tao ${ }^{1}$, Fenglei $\mathrm{Xu}^{2}$, Yanling $\mathrm{Hu}^{1,3^{*}}$ (D), Chengdong Zhang ${ }^{1}$ and Guangjie Zhou ${ }^{1}$
}

\begin{abstract}
Background: Due to the intraarticular and complex nature of the coronal shear fracture of the humeral capitellum and its rarity, it has been difficult to formulate a universally accepted method of surgical management. The purpose of this study is to retrospectively evaluate the clinical outcomes of 15 patients with isolated coronal shear fractures of the capitellum treated by Herbert screw fixation through anterolateral approach, and to address the safety and tips for this surgical procedure.
\end{abstract}

Methods: This retrospective study included 15 isolated coronal shear fractures of the capitellum without posterior involvement, which were classified according to the Dubberley classification as 11 type $1 \mathrm{~A}$ fractures and 4 type $3 \mathrm{~A}$ fractures. All fractures were treated with Herbert screws fixation via the anterolateral approach. Clinical and radiographic evaluation was performed regularly, with a mean follow-up of 29 months.

Results: The mean operative time was 81 min. There were no wound healing problems or infection. One incomplete posterior interosseous nerve injury occurred, which recovered soon without residual compromise. All fractures healed well. At the final follow-up, the average range of motion was $134^{\circ}$ in flexion-extension and $172^{\circ}$ in supination-pronation. There was no significant difference between the affected and the unaffected elbows with regard to motion in flexion-extension or flexion-extension. The average Mayo Elbow Performance Index Score was 93 with 11 excellent and 4 good. No evidence of avascular necrosis, posttraumatic osteoarthritis, or heterotrophic ossification was found.

Conclusion: Open reduction and internal fixation using Herbert screws through a anterolateral approach is a reliable and effective treatment for coronal shear fractures of capitellum, and able to achieve stable fixation and restoration of a functional range of motion.

Keywords: Capitellum, Fracture, Internal fixation, Anterolateral approach, Herbert screw

\section{Background}

Fractures of the humeral capitellum are rare injuries, accounting for nearly $1 \%$ among all elbow fractures [1]. These fractures are usually a result of axial loading to the capitellum and occasionally to the trochlea transmitted through the radial head.

\footnotetext{
* Correspondence: huyanlingqy@126.com

'Department of Orthopaedic Surgery, Affiliated Hospital of Qingdao University, Qingdao, Shandong 266000, People's Republic of China ${ }^{3}$ Department of Orthopaedic Surgery, Affiliated Hospital of Qingdao University, 59 Haier Road, Qingdao City 266003, Shandong Province, China Full list of author information is available at the end of the article
}

Treatment strategies for these injuries have evolved from conservative management to surgical management. Currently, open reduction and internal fixation with an aim to provide stable and congruent joint has been regarded as preferred treatment [2-8], whereas the intraarticular and complex nature of these fractures makes optimal surgical exposure and fixation method debatable. For coronal shear fracture of capitellum without involvement of posterior aspect, the most commonly used approach is the lateral approach of elbow joint [2-8]. Though favorable outcomes have been reported, exposure of the radiocapitellar 
compartment and visualization of the trochlea and medial articular extension is inadequate through this approach. Several studies have adopted the anterolateral approach to the elbow joint to treat this type of fracture, which is superior to the lateral approach for exposure range of the anterior aspect of the elbow joint [9-11]. As regard to articular surface reconstruction, various implants including Kirschner wires, headleass compression screws, Herbert screws, minifragment screws, and bioabsorbable implants have been adopted. Herbert screw fixation is a good option due to excellent compression at the fracture site, stable fixation, and nonprominence of the implant intra-articularly [12].

Given the intraarticular and complex nature of the coronal shear fracture of the humeral capitellum and its rarity, it has been difficult to formulate a universally accepted method of surgical management. The purpose of this study is to present the clinical outcomes of a retrospective study of 15 cases with isolated coronal shear fractures of the capitellum treated by Herbert screw fixation through anterolateral approach, and to address the safety and tips for this surgical procedure. We hypothesized that open reduction and Herbert screws fixation through anterolateral approach is a reliable and effective management for coronal shear fractures of capitellum.

\section{Methods}

\section{Patients}

From January 2009 to June 2015, 18 consecutive patients with isolated coronal shear fractures of the capitellum without posterior condyle involvement were treated with open reduction and Herbert screw fixation through the anterolateral approach within 2 weeks after the injury. Two patients were lost to follow-up, and 1 died of unrelated illness. This study was approved by the Institutional Review Board of the affiliated hospital of Qingdao University, and 15 patients consented to participate in the study. Nine patients were female, and 6 were male. The mean age (and standard deviation) was $42 \pm 13$ years (range, 19 to 64 years). Ten patients occurred after a fall, and 5 occurred in road traffic accidents. The mean time from presentation to surgical treatment was $4 \pm 1$ days (range, 1 to 7 days). The mean duration of follow-up was $29 \pm 4$ months (range, 24 to 36 months) (Table).

Plain radiographs were performed routinely. All patients underwent computed tomography $(\mathrm{CT})$ scans with a $3 \mathrm{D}$ reconstruction for better definition of the fracture line and to rule out associated injuries, such as the coronoid process fractures, a dislocation or injury to the radial head, epicondylar avulsion fractures or elbow dislocations. Only isolated coronal shear fractures were included in this study.

Fractures in this study were classified according to the Dubberley classification system [2].Type 1 is a fracture involving primarily the capitellum with or without the lateral trochlear ridge. Type 2 is a fracture involving the capitellum and the trochlea as one piece. Type 3 is a fracture involving both the capitellum and the trochlea as separate fragments. These fractures were further classified as type A and type B based on the absence or presence of posterior condylar comminution. All fractures in the current series were coronal shear fractures without posterior comminution. Eleven patients sustained type $1 \mathrm{~A}$ fracture, 4 were type $3 \mathrm{~A}$.

\section{Surgical technique}

All the patients were administered a brachial plexus anesthesia and placed in the supine position with a tourniquet on the upper arm. Varus and valgus stress examination under anesthesia was performed to rule out concomitant ligamentous injury.

A curved incision began $5 \mathrm{~cm}$ above the elbow flexion crease in the supinated forearm, and followed the lateral border of the biceps distally, but curved laterally at the elbow joint level to avoid crossing a flexion crease at $90^{\circ}$. Then it extended distally in the forearm along the medial border of brachioradialis. The interval was made between the brachialis and brachioradialis. The forearm lateral cutaneous nerve need to be protected in the superficial plane. In the deeper plane, the radial nerve need to be identified and protected (Fig. 1a). The brachioradialis and the radial nerve were retracted laterally and the biceps medially to expose the anterior capsule of the elbow joint. The capsule was incised to expose the capitellum (Fig. 1b).

The fracture site was debrided by removing blood clots, loose pieces of bone, and any interposed tissue. Saline irrigation was used to achieve greater clarity. The fracture was reduced by matching the articular fracture lines. Provisional fixation was performed with 2 or 3 guidewires for the Herbert screw. The guidewires were passed across the fracture site where the planned screw track was to be inserted. After anatomic reduction was confirmed with fluoroscopy, Herbert screws were inserted over the guidewires in anterior to posterior direction to achieve definitive fixation. The screws were buried beneath the articular surface. Upon fixation, the elbow was made to go through the full flexion-extension and rotation arc to check for the stability of fixation. Final reduction and position of the implant was checked with fluoroscopy. The closure of the wound was done in layers over a drain.

\section{Postoperative care}

A long arm posterior plaster splint was applied routinely with the elbow at approximately $90^{\circ}$ of flexion, which was kept for 2 week. Active range of motion (ROM) was started when the splint was removed. 


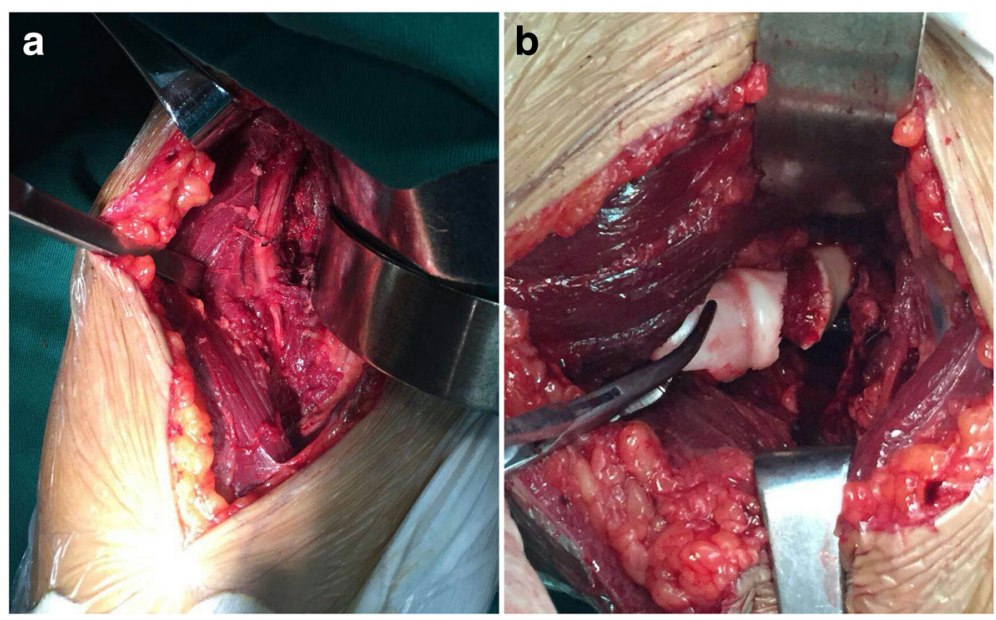

Fig. 1 Dissection of anterolateral approach to the elbow joint. a: The dissection of radial nerve. $\mathbf{b}$ : The exposure of fracture fragment

Operation time, wound healing complication and nerve injury were recorded. Clinical and radiographic evaluation was performed postoperatively at approximately 1, 2, 3, 6, 12 months. Then patients were called back for evaluation for the purposes of this study. At each follow-up, Pain, ROM and stability of the elbow joint was assessed by clinical examination, which enabled calculation of the Mayo Elbow Performance Index (MEPI) Score. The MEPI consists of four parts: pain (with a maximum score of 45 points), ulnohumeral motion (20 points), stability (10 points) and the ability to perform five functional tasks ( 25 points). If the total score is included between 90 and 100 points, it can be considered excellent; between 75 and 89 points, good; between 60 and 74 points, fair; and less than 60 points, poor. In addition, radiographic examination was performed to evaluate the status of the bony union, heterotopic ossification, incidence of posttraumatic osteoarthritis and avascular necrosis.

\section{Statistical analysis}

The paired t-test was used for statistical comparisons with regard to ROM between the affected and the unaffected elbow with SPSS for Windows 15.0 (SPSS, USA). Differences were considered to be significant if $p<0.05$.

\section{Results}

The mean operative time was $81 \pm 12$ min (range, 65 to $105 \mathrm{~min}$ ). There were no wound healing problems and infection. One patient sustained incomplete posterior interosseous nerve palsy, who presented with extension deficit of his ring finger and little finger at the metacarpophalangeal joint level. It recovered completely in 4 weeks without residual compromise. All fractures healed well in their normal anatomic position as seen on radiographs.
At the final follow-up, three patients reported mild pain and one described moderate ache during activity without restriction in activities of daily living. No patient had any subjective complaints of instability of the elbow. The average loss of ROM of the affected elbows was $10^{\circ}$ of flexionextension and $7^{\circ}$ of supination-pronation compared with the unaffected elbows. But the average ROM of the affected and unaffected elbows did not differ significantly with respect to flexion-extension $\left(134^{\circ} \pm 10^{\circ}\right.$ and $144^{\circ} \pm 4^{\circ}$ respectively; $p=0.066)$, and supination-pronation $\left(172^{\circ} \pm 11^{\circ}\right.$ and $179^{\circ} \pm 2^{\circ}$ respectively; $p=0.083$ ). The average MEPI Score was $93 \pm 8$ (range, 75 to 100) with 11 excellent and 4 good. All patients were satisfied with the operative outcome and returned to their previous activity levels. No evidence of avascular necrosis, posttraumatic osteoarthritis, or heterotrophic ossification was found (Table 1). An illustrative case was shown in Fig. 2.

\section{Discussion}

Surgical approaches are usually based on fracture type and complexity, comfort of the orthopedic surgeon, and protection of the blood supply. For coronal shear fracture of capitellum without posterior condyle involvement, the surgical approaches include the lateral approach and anterolateral approach to the elbow joint. The most commonly used approach in the literature is the lateral approach, which allows exposure to the elbow joint by elevation of the common extensor origin from the lateral epicondyle [2-8]. Nevertheless, its disadvantage is inadequate exposure of the capitulum and trochlea, which can limit visualization of accurate reduction of the fracture fragment, and make it relatively difficult to insert the screws perpendicular to the fracture site, especially for those capitellar fractures that extend to the trochlea. Dubberley et al. suggested that a flexor-pronator split or sectioning of the lateral collateral ligament should be performed if the medial aspect of the trochlea can not 
Table 1 Demographics and clinical outcomes of reported patients

\begin{tabular}{|c|c|c|c|c|c|c|c|c|}
\hline Patient & Gender & $\begin{array}{l}\text { Age } \\
\text { (year) }\end{array}$ & Mechnism & $\begin{array}{l}\text { Dubberley } \\
\text { classification }\end{array}$ & $\begin{array}{l}\text { Follow-up } \\
\text { (month) }\end{array}$ & $\begin{array}{l}\text { ROM in flexion / } \\
\text { extension (degree) }\end{array}$ & $\begin{array}{l}\text { ROM in supination / } \\
\text { pronation (degree) }\end{array}$ & MEPI Score \\
\hline 1 & $F$ & 26 & Fall & $1 \mathrm{~A}$ & 24 & 145 & 180 & 100 \\
\hline 2 & M & 48 & Fall & $1 \mathrm{~A}$ & 30 & 140 & 180 & 95 \\
\hline 3 & $\mathrm{~F}$ & 64 & RTA & $3 \mathrm{~A}$ & 36 & 130 & 160 & 85 \\
\hline 4 & $\mathrm{~F}$ & 35 & Fall & $1 \mathrm{~A}$ & 34 & 135 & 180 & 100 \\
\hline 5 & M & 51 & RTA & $3 \mathrm{~A}$ & 28 & 125 & 155 & 85 \\
\hline 6 & M & 28 & Fall & $1 \mathrm{~A}$ & 36 & 145 & 180 & 100 \\
\hline 7 & $\mathrm{~F}$ & 52 & RTA & $3 A$ & 31 & 120 & 155 & 85 \\
\hline 8 & M & 19 & RTA & $1 \mathrm{~A}$ & 33 & 150 & 180 & 100 \\
\hline 9 & $F$ & 56 & Fall & $1 \mathrm{~A}$ & 29 & 130 & 175 & 95 \\
\hline 10 & M & 60 & Fall & $1 \mathrm{~A}$ & 25 & 125 & 160 & 90 \\
\hline 11 & $\mathrm{~F}$ & 43 & Fall & $1 \mathrm{~A}$ & 24 & 130 & 175 & 95 \\
\hline 12 & $\mathrm{~F}$ & 39 & Fall & $1 \mathrm{~A}$ & 26 & 130 & 180 & 95 \\
\hline 13 & $\mathrm{~F}$ & 57 & Fall & $3 A$ & 27 & 120 & 155 & 75 \\
\hline 14 & $\mathrm{~F}$ & 33 & Fall & $1 \mathrm{~A}$ & 24 & 145 & 180 & 100 \\
\hline 15 & M & 23 & RTA & $1 \mathrm{~A}$ & 24 & 145 & 180 & 100 \\
\hline
\end{tabular}

ROM range of motion, MEPI Mayo Elbow Performance Index, $F$ female, Fall ground level fall, $M$ male, RTA road traffic accident

be seen adequately or reduction can not be confirmed from the lateral approach [2]. The anterolateral approach can expose the capitellum and trochlea widely enough to facilitate the reduction and fixation of the intra-articular fragments by directly approaching the anterior aspect of the elbow [9-11]. In addition, the anterolateral approach avoids the release of the common extensor origin, which may lead to postoperative extensor lag. So the use of anterolateral approach can circumvent the disadvantages of lateral approach. Based on our experience, the anterolateral approach was characterized by sufficient visualization of the joint including the medial articular surface, ease of achieving anatomic reduction and perpendicular fixation with screws in anterior to posterior direction.

The disadvantage for anterolateral approach is the plane of dissection much closer to the important neurovascular structures in the elbow, which carries a risk of iatrogenic injury to the radial nerve. In two research reports about anterolateral approach, no nerve injury occurred. Whereas Vaishya R et al. [11] reported one patient in their case series sustained postoperative posterior interosseous nerve palsy that recovered completely. In this case series, one incomplete posterior interosseous nerve injury occur, which recovered soon without residual compromise. In our opinion, the radial nerve dissected in this approach can be easily retracted and protected. The incidence of radial nerve injury is very small with direct visualization and careful retraction.

Fractures of elbow are usually associated with ligamentous injuries which can lead to elbow instability. Coronal shear fractures of the capitellum combined with elbow dislocation have been reported by Giannicola et al. as a potential pattern of complex elbow instability

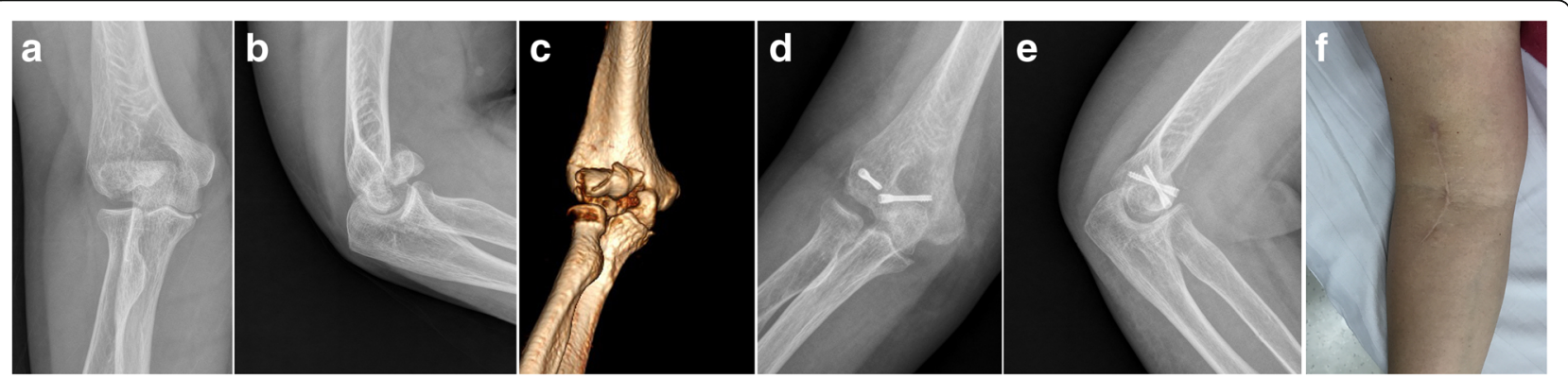

Fig. 2 A 64-year-old female with type 3A capitellar fracture surgically treated with open reduction and Herbert screw fixation through anterolateral approach. $\mathbf{a}$ and $\mathbf{b}$ : Anteroposterior and lateral x-ray views of the fracture preoperatively. $\mathbf{c}$ : 3D CT reconstruction of the fracture preoperatively. The fragments were displaced anteriorly and superiorly. $\mathbf{d}$ and $\mathbf{e}$ : Anteroposterior and lateral x-ray views 12 months postoperatively showed union of the capitellar fracture which was fixed with 2 Herbert screws in an anterior to posterior fashion. $\mathbf{f}$ : Incision appearance of anterolateral approach 
where eventual associated injuries of the lateral collateral ligament (LCL) and medial collateral ligament (MCL) should be assessed [13]. In the study of Mighell et al., Dubberley type 1A capitellar fractures were not associated with LCL injury, and two LCL injuries were found in five type 2A capitellar fractures [3]. Are A et al. reported no evidence of any LCL or MCL injury for isolated type 1A capitellar fractures [5]. In this series, no concomitant LCL or MCL injuries were found. In our opinion, ligamentous injuries occur in capitellar fractures combined with elbow dislocations. Examination under anesthesia should be done as a routine to rule out potential ligamentous instability. For coronal capitellar fractures with LCL injury diagnosed intraoperatively, lateral approach is preferred because the LCL injury and fracture can be treated through one lateral incision.

The method of fracture fixation is also an issue of interest for fracture management. Kirschner wires, metallic screws, bioabsorbable implants and fibrin glue have been reported for reconstruction of capitellar fractures [12]. Metallic screw fixation was the most commonly used technique. Biomechanical studies have demonstrated that metallic screws can provide favourable stability for constructed capitellum fractures [14, 15]. Although several different types of screw (cannulated, cortical lag, cancellous, headless, and Herbert screws) were used, no direct comparison between the different screws could be made due to the heterogeneous reporting of clinical outcomes. Nowadays, Herbert screws fixation has become popular for capitellar fractures and good clinical results have been published because the advantages offered by these screws include excellent compression at the fracture fragments, stable fixation, and nonprominence of the implant intraarticularly $[7,16]$.

In the present study, good-to-excellent outcomes were achieved according to MEPI Score for all the shear fractures of capitellum without involvement of posterior aspect. These results were similar to those previously published [4, 6, 17]. Although four patients reported mild or moderate pain during activity, all patients were satisfied with the operative outcomes and returned to their previous levels of activity. Furthermore, no avascular necrosis or posttraumatic osteoarthritis occurred in this case series, which is in accordance with several studies $[5,7,9-11]$. In our opinion, protection of capitellar soft tissue attachment, anatomical reduction and stable fixation with minimal damage to articular cartilage can minimize the incidence of both complications. In general, satisfactory outcomes for coronal shear fractures of capitellum can be expected by accurate reduction, adequate fixation and early functional exercises. All of these can be easily achieved by the use of Herbert screw fixation through anterolateral approach to the elbow joint.
The limitation of this study was the small number of patients and short-term follow-up period. The larger numbers of patients and longer follow-up period is necessary to determine the true incidence of osteonecrosis and posttraumatic arthritis.

\section{Conclusion}

Our findings suggest that open reduction and Herbert screw internal fixation through the anterolateral approach is a reliable and effective treatment for coronal shear fractures of capitellum, and able to achieve stable fixation and restoration of a functional range of motion.

\section{Abbreviations}

LCL: Lateral collateral ligament; MCL: Medial collateral ligament; MEPI: Mayo elbow performance index; ROM: Active range of motion

\section{Acknowledgements}

We would like to thank all the staff in Department of Orthopaedic Surgery, Affiliated Hospital of Qingdao University for their help on this study.

\section{Funding}

We received no external funding for this study.

\section{Availability of data and materials}

The full dataset is available from the first and corresponding authors upon request.

\section{Authors' contributions}

TB Y, YL H, HT and FL X made substantial contributions to the design of this study, acquisition of data, analysis and interpretation of data, and drafting of the manuscript; CD Z and GJ Z participated in the design of the study, and assisted with drafting the manuscript. All authors read and approved the final manuscript.

\section{Ethics approval and consent to participate}

This study was approved by the Institutional Review Board of the affiliated hospital of Qingdao University. Written informed consent was obtained from all patients enrolled in the investigation.

\section{Consent for publication}

Not applicable.

\section{Competing interests}

The authors declare that they have no competing interests.

\section{Publisher's Note}

Springer Nature remains neutral with regard to jurisdictional claims in published maps and institutional affiliations.

\section{Author details \\ 'Department of Orthopaedic Surgery, Affiliated Hospital of Qingdao University, Qingdao, Shandong 266000, People's Republic of China. ${ }^{2}$ Department of Radiology, Affiliated Hospital of Qingdao University, Qingdao, Shandong 266000, People's Republic of China. ${ }^{3}$ Department of Orthopaedic Surgery, Affiliated Hospital of Qingdao University, 59 Haier Road, Qingdao City 266003, Shandong Province, China.}

Received: 5 December 2017 Accepted: 23 March 2018

Published online: 05 April 2018

\section{References}

1. Bryan RS, Morrey BF. Fractures of the distal humerus. In: Morrey BF, edtior. The elbow and its disorders. 3rd Philadelphia, PA: WB Saunders; 1985. p 325-333. 
2. Dubberley JH, Faber KJ, Macdermid JC, Patterson SD, King GJ. Outcome after open reduction and internal fixation of capitellar and trochlear fractures. J Bone Joint Surg Am. 2006;88(1):46-54.

3. Mighell M, Virani NA, Shannon R, Echols EL Jr, Badman BL, Keating CJ. Large coronal shear fractures of the capitellum and trochlea treated with headless compression screws. J Shoulder Elb Surg. 2010;19(1):38-45.

4. Ruchelsman DE, Tejwani NC, Kwon YW, Egol KA. Open reduction and internal fixation of capitellar fractures with headless screws. J Bone Joint Surg Am. 2008;90(6):1321-9.

5. Are A, Tornatore I, Theodorakis E. Operative management of a shear fracture of the bilateral capitellum: a case report and review of the literature. Chin J Traumatol. 2016;19(4):231-4.

6. Ashwood N, Verma M, Hamlet M, Garlapati A, Fogg Q. Transarticular shear fractures of the distal humerus. J Shoulder Elb Surg. 2010;19(1):46-52.

7. Singh AP, Singh AP, Vaishya R, Jain A, Gulati D. Fractures of capitellum: a review of 14 cases treated by open reduction and internal fixation with Herbert screws. Int Orthop. 2010;34(6):897-901.

8. Patterson SD, Bain Gl, Mehta JA. Surgical approaches to the elbow. Clin Orthop. 2000;370:19-33.

9. Cornelius AL, Bowen TR, Mirenda WM. Anterolateral approach for an unusual pediatric capitellar fracture: a case report and review of the literature. lowa Orthop J. 2012:32:215-9.

10. Imatani J, Morito $Y$, Hashizume $H$, Inoue $H$. Internal fixation for coronal shear fracture of the distal end of the humerus by the anterolateral approach. Shoulder Elbow Surg. 2001;10(6):554-6.

11. Vaishya R, Vijay V, Jha GK, Agarwal AK. Open reduction and internal fixation of capitellar fracture through anterolateral approach with headless doublethreaded compression screws: a series of 16 patients. J Shoulder Elb Surg. 2016;25(7):1182-8

12. Trinh $T Q$, Harris JD, Kolovich GP, Griesser MJ, Schickendantz MS, Jones GL. Operative management of capitellar fractures: a systematic review. J Shoulder Elb Surg. 2012;21(11):1613-22.

13. Giannicola G, Sacchetti FM, Greco A, Cinotti G, Postacchini F. Management of complex elbow instability. Musculoskelet Surg. 2010;94(Suppl 1):S25-36.

14. Koslowsky TC, Zilleken C, Dargel J, Thelen U, Burkhart KJ, Heck S, et al. Reconstruction of a Bryan and Morrey type I capitellar fracture in a sawbone model with four different fixation devices: an experimental study. Injury. 2012;43(3):381-5.

15. Elkowitz SJ, Polatsch DB, Egol KA, Kummer FJ, Koval KJ. Capitellum fractures: a biomechanical evaluation of three fixation methods. J Orthop Trauma. 2002;16(7):503-6.

16. Sano S, Rokkaku T, Saito S, Tokunaga S, Abe Y, Moriya H. Herbert screw fixation of capitellar fractures. J Shoulder Elb Surg. 2005;14(3):307-11.

17. Tarallo L, Mugnai R, Adani R, Zambianchi F, Costanzini CA, Catani F. Shear fractures of the distal humerus: is the use of intra-articular screws a safe treatment? Musculoskelet Surg. 2015;99(3):217-23.

\section{Submit your next manuscript to BioMed Centra and we will help you at every step:}

- We accept pre-submission inquiries

- Our selector tool helps you to find the most relevant journal

- We provide round the clock customer support

- Convenient online submission

- Thorough peer review

- Inclusion in PubMed and all major indexing services

- Maximum visibility for your research

Submit your manuscript at www.biomedcentral.com/submit

CBiomed Central 